\title{
Altered Expression of Oxidative Metabolism Related Genes in Cholangiocarcinomas
}

\author{
Ratchadawan Aukkanimart ${ }^{1,2,3}$, Thidarut Boonmars ${ }^{1,2 *}$, Amornrat Juasook ${ }^{2,4}$, \\ Pranee Sriraj ${ }^{1,2,3}$, Sirintip Boonjaraspinyo ${ }^{1,2,5}$, Zhiliang $\mathrm{Wu}^{6}$, Porntip \\ Laummuanwai ${ }^{1}$, Chawalit Pairojkul ${ }^{7}$, Narong Khuntikeo ${ }^{8}$, Panaratana \\ Rattanasuwan 9
}

\begin{abstract}
Cholangiocarcinoma (CCA) is a rare but highly fatal cancer for which the molecular mechanisms and diagnostic markers are obscure. We therefore investigated the kinetic expression of isocitrate dehydrogenase-1 (IDH1), isocitrate dehydrogenase-2 (IDH2) and homogentisate 1,2-dioxygenase (HGD) during the tumorigenesis of $O$. viverrini infection-associated CCA in an animal model, and confirmed down-regulation of expression in human cases of opisthorchiasis-associated CCA through real time PCR. Kinetic expression of HGD, IDH1 and IDH2 in the animal model of $O$. viverrini infection-induced CCA was correlated with human CCA cases. In the animal model, expression of HGD was decreased at all time points $(p<0.01)$ and expression of both IDH1 and IDH2 was decreased in the CCA group. In human cases, expression of HGD, IDH1 and IDH2 was decreased more than 2 fold in 55 cases $(70.5 \%), 25$ cases $(32.1 \%)$ and 24 cases $(30.8 \%)$ respectively. The present study suggests that reduction of HGD, IDH1 and IDH2 may be involve in cholangiocarcinoma genesis and may be useful for molecular diagnosis.
\end{abstract}

Keywords: Liver fluke - cholangiocarcinogenesis - (IDH1) - IDH2 - (HGD) - oxidative metabolism related genes

Asian Pac J Cancer Prev, 16 (14), 5875-5881

\section{Introduction}

Infection of Opisthorchis viverrini can induce tumorigenesis of cholangiocarcinoma (CCA), which has been demonstrated by the epidemiological investigations and animal experiments (Thamavit et al., 1987:1983; IARC, 1994; Sithithaworn et al., 2003; Sriamporn et al., 2004; IARC, 2011). As one of the risk factors, combining local traditional eating custom of fermented food which contains potent carcinogen, for example, $\mathrm{N}$-nitrosodimethylamine (NDMA), opisthorchiasisassociated CCA has become a public health problem in endemic region, especially in the northeastern of Thailand where the highest prevalence of CCA in the world was observed (Jongsuksuntigul et al., 2003; Sriamporn et al., 2004; Sripa et al., 2008). Many efforts have been made to reveal the mechanism of tumorigenesis of CCA induced by $O$. viverrini infection. Although it is not still unknown completely, NOS derived from inflammation caused by the infection is considered to be one of factors to cause the tumorigenesis. It has been demonstrated that $O$. viverrini caused the accumulation of NOS (Srianujata et al., 1984; Srivatanakul et al., 1991; Pinlaor et al., 2004b). The accumulated NOS may induce the DNA damage, such as, epigenetic changes and genomic instability, which may initial tumorigenesis. The oxidative DNA damage was observed during $O$. viverrini infection, for example, 8-nitroguanine and 8-oxo-7,8-dihydro-20deoxyguanosine (8-oxodG), an indicator of oxidative DNA damage, mediating iNOS dependent DNA damage in intrahepatic bile duct epithelium (Pinlaor et al., 2004a; Kawanishi et al., 2006). Under a physiological condition, there is a constant endogenous production of ROS. To control the balance between production and removal of ROS, there are a series of protective molecules, defined as antioxidant defenses, including superoxide dismutase, catalase, glutathione peroxidase and glutathione-Stransferase, glutathione, cysteine and thioredoxin etc (McCord et al., 1969; Chelikani et al., 2004; Hayes et al., 2005; Valko et al., 2007; Raza, 2011; Miki \& Funato, 2012). Our previous cDNA microarray indicated that in the animal model of $O$. viverrini infection-induced CCA,

\footnotetext{
${ }^{1}$ Department of Parasitology, ${ }^{2}$ Liver Fluke and Cholangiocarcinoma Research Center, ${ }^{5}$ Department of Community Medicine, ${ }^{7}$ Department of Pathology, ${ }^{8}$ Department of Surgery, ${ }^{9}$ Department of Anesthesiology, Faculty of Medicine, Khon Kaen University, Khon Kaen, ${ }^{3}$ Rajamangala University of Technology Isan Sakonnakhon Campus, Sakonnakhon, ${ }^{4}$ Faculty of Veterinary Medicine, Mahasarakham University, Mahasarakham, Thailand, ${ }^{6}$ Department of Parasitology, Graduate School of Medicine, Gifu University, Gifu, Japan*For correspondence: bthida@kku.ac.th; boonmars@yahoo.com
} 
the expressions of many antioxidative enzyme related genes were down-regulated, for example, IDH (isocitrate dehydrogenase), HGD (homogentisate 1,2-dioxygenase), Gsta (glutathione S-transferase), GST (glutathione synthetase) and Prdx6 (peroxiredoxin 6) (Wu et al., 2011). Therefore, we speculate that the down-regulated expressions of these antioxidative enzymes may play the important roles in the tumorigenesis of opisthorchiasisassociated CCA.

The IDH1 and IDH2 are the enzymes to catalyze the oxidative decarboxylation of isocitrate to alphaketoglutarate, contributing to NADPH production by generating NADPH from NADH+. NADPH is required for the synthesis of glutathione, which protects cells from redox stress. HGD is one of the enzymes required for the catabolism of the aromatic amino acids phenylalanine and tyrosine, and has oxidoreductase activity to catalyze oxidation-reduction (redox) reaction. So far, most of the studies on IDH were focused to the mutation of the genes in gliomas, the tumors of central nervous system (Hartmann et al., 2009; Qi et al., 2011; Kim et al., 2012). Little has been reported on the down-regulation of IDH in other cancers and its relationship with tumorigenesis, and a few reports are found on these enzymes in human $O$. viverrini related and non- $O$. viverrini related CCAs (Chan-On et al., 2013; Jiao et al., 2013). Therefore, in the present study, we investigated the kinetic expression of IDH1, IDH2 and HGD during the tumorigenesis of $O$. viverrini infection-induced CCA in animal model, and confirmed the gene regulation of the expression in the human CCA in endemic area of $O$. viverrini infection.

\section{Materials and Methods}

\section{Parasite and infection}

An animal model for $O$. viverrini infection-induced CCA has been well established (Thamavit et al., 1987:1993). Infection only or feeding NDMA-containing water only does not cause formation of CCA, while infection plus feeding NDMA-containing water can induce the development of CCA.

Metacercariae of $O$. viverrini were collected as the previously described (Boonmars et al., 2008; Boonmars et al., 2009a; Boonmars et al., 2011). In brief, naturally infected cyprinoids fish captured from a fresh water reservoir in an endemic area of Khon Kaen, Northeast Thailand, were minced and digested with pepsin- $\mathrm{HCl}$, filtrated and then washed with saline. Afterwards the Opisthorchis metacercaria which was 204 x $145 \mu \mathrm{m}$, oval-shaped, with large, black excretory bladders - were identified under a dissecting microscope.

Eighty Syrian hamsters (6 weeks) were divided in to 4 groups ( $\mathrm{n}=20$ per group): (1) normal group; (2) infection group: 20 hamsters were infected with 50 metacercariae; (3) NDMA group: 20 hamsters were maintained with drinking water containing $12.5 \mathrm{ppm}$ of NDMA (Wako, Japan) for 2 months; and (4) infection plus NDMA group: 20 hamsters were infected with 50 metacercariae and maintained with drinking water containing $12.5 \mathrm{ppm}$ of NDMA for 2 months. Hamster livers (five hamsters at each time point for each group) were collected at 1,2, 3 and 6 month(s) post infection (p.i.) for total RNA isolation and histological observation.

\section{Histopathological changes}

The hamster liver of each group was processed as the previous study (Boonmars et al., 2011). In brief, liver was fixed with $10 \%$ neutral formalin and cleared with xylene. And then was paraffinized embedded. Liver sections were deparaffinized in xylene, rehydrated through a descending alcohol series, washed with distilled water and stained with Harris's hematoxylin followed by eosin. Finally, the sections were dehydrated through an alcohol series, cleared in xylene, and mounted on slides using Permount resin. The liver sections were observed and digitized under a light microscope (Olympus BX51; Tokyo, Japan) for pathological grading.

\section{Collection of human CCA and adjacent tissues}

Seventy eight pairs of liver samples from CCA patients coming from heavy epidemical area of opisthorchiasis were provided by the Liver Fluke and Cholangiocarcinoma Research Center, Faculty of Medicine, Khon Kaen University, Thailand. Each pair of sample included the CCA tumor tissue and the adjacent normal tissues from same patient were histologically confirmed. The clinicopathological features of the CCA patients were represented by status, histological type, histological grade, stage and metastasis based on the criteria of the Liver Cancer Study Group of Japan, and the International Union Against Cancer (UICC)/American Joint Committee on Cancer (AJCC). The samples were defined pathologically and histologically (Table 1). Total RNA was isolated from the tumor and adjacent normal tissues, and subjected to the real time PCR for the quantity of expression level as described below. The utilization of the specimens in the present study was approved by the Human Ethics Committee of the Khon Kaen University (Ethical Clearance No. HEKKU501153) and Gifu University.

\section{RNA isolation}

Total RNA was extracted from the tissues $(200 \mathrm{mg}$ ) at the same region (hilar region of liver) from the four groups mentioned above (normal, infection, NDMA and infection plus NDMA) using TRIZOL (Invitrogen, Carlsbad, CA, USA) according to the manufacturer instructions. The isolated RNA was treated with DNase (RQ1 RNase-Free DNase, Promega, Co., Madison, WI, USA). The treated RNA was purified using conventional method and dissolved in RNase-free water.

\section{Quantitative real time PCR}

Quantitative real-time RT-PCR was performed to determine the expressions of IDH1, IDH2 and HGD using the Thermal Cycler Dice Real Time System (TAKARA, Japan). Because there was not genetic information on hamster IDH1 and IDH2, we first determined the sequences of hamster of these two genes with the primers developed based on the mouse sequence, and then designed the primers for hamster IDH1 (forward ggttatggttccettggtatga and reverse gatgtctcctgtcctttctggta), IDH2 (forward tgccetgatgggaagacaa and reverse gcaaagatgctggcgatagg) 
and HGD (forward ttgcttccaaacaggatgtctc and reverse atggtcaaaggccactgcat). The primer for hamster G3PDH (forward gacatcaagaaggtggtgaagca and reverse catcaaaggtggaagagtggga) was designed based on the previous published paper (Boonjaraspinyo et al., 2012).

The primers for human IDH1, IDH2, HGD and GUSB were designed based on the published sequences in GenBank, IDH1: forward ggaacccaaaaggtgacatacc and reverse caaactgggacttgtactgcttg; IDH2: forward caaaatggtcttcacccaaa and reverse catcgtaggctttcagtatggt; HGD: forward gatgetgactgetttgagaagg and reverse cactttgtgaccgccagactta and GUSB: forward atggaagaagtggtgcgtacg and reverse aaggatttggtgtgagcga.

Total RNA was isolated and purified as mentioned above. Reverse transcription was performed using a Prime Reverse Transcriptase (TAKARA BIO Inc., Ootsu, Shiga, Japan) according to the manufacturer's instructions. Real time PCR was performed as previous described (Boonmars et al., 2009b). In brief, optimal conditions for all investigated genes were established using SYBR Premix Ex Taq Kit (TAKARA BIO) according to manufacturer's instructions. Twenty $\mu$ l of the reaction solution consisted of $2 \mu \mathrm{l}$ of the template (appropriate dilution was determined by genes), $10 \mu$ l of SYBR Premix Ex Taq, $0.8 \mu \mathrm{l}$ of $5 \mu \mathrm{M}$ of each primer. PCR amplification was performed as follows: predenature for 1 cycle at 95 ${ }^{\circ} \mathrm{C}$ for $30 \mathrm{~s}$, and 40 cycles at $95{ }^{\circ} \mathrm{C}$ for $5 \mathrm{~s}, 60-62{ }^{\circ} \mathrm{C}$ for 30s. Melting curve analysis was done at $65^{\circ} \mathrm{C}$ to $95^{\circ} \mathrm{C}$ with $0.1^{\circ} \mathrm{C} /$ second temperature transition.

Specific external controls were constructed for target genes. The PCR fragment of each gene was cloned into a pT7Blue T-Vector (Novagen, Inc., Madison, WI, USA). The recombinant plasmids were introduced into competent cells of Escherichia coli JM 109. The plasmid DNA was isolated from E. coli using a FlexiPrep Kit (Amersham
Pharmacia Biotech Inc., Piscataway, NJ, USA). Ten-fold serial dilutions (101 to 107 copies/ $2 \mu \mathrm{l}$ ) of the plasmids were used to generate standard curves for each gene.

Differences in the amount of cDNA from different samples were normalized by quantification of the housekeeping gene G3PDH and expression levels were represented as the copy number of target gene /106 $\mathrm{G} 3 \mathrm{PDH}$ copies. The values are expressed as mean \pm SD.

\section{Results}

\section{Histopathology}

The histological changes was similar to our previous study (Boonmars et al., 2011). In brief, the OV infected group was observed the inflammatory cell aggregation at all-time points (1,2,3 and 6 months) of observation. The NDMA treatment group was observed the inflammatory cell aggregation at all-time points and bile duct proliferation at 2 and 3 months and cholangiocarcinoma at 6 months. The group of infection plus NDMA treatment was observed inflammatory cell aggregation at all-time points of observation. Bile duct proliferation was observed at 2 and 3 months and the CCA area was observed at 6 months.

Kinetic expression of HGD, IDH1 and IDH2 in the animal model of $O$. viverrini infection-induced CCA

The expression kinetics of HGD, IDH1 and IDH2 during the development of CCA was determined by real time PCR, in the hamster groups of normal, infection only, NDMA-containing water feeding only, and infection plus NDMA-containing water feeding at 1, 2, 3 and 6 months after infection. As shown in (Figure 1), infection only caused down-regulation of HGD expression. The expression of HGD was decreased at 1 month p.i. $(p<0.05)$, lowest at 2 months p.i. $(p<0.01)$, and returned

Table 1. The Correlation of Expressions of HGD, IDH1 and IDH2 with Clinicopathology in 78 CCA Patients

\begin{tabular}{|c|c|c|c|c|c|c|c|c|c|c|}
\hline \multicolumn{2}{|c|}{ Clinicopathological parameter } & \multicolumn{3}{|c|}{ Expression of HGD mRNA } & \multicolumn{3}{|c|}{ Expression of IDH1 mRNA } & \multicolumn{3}{|c|}{ Expression of IDH2 mRNA } \\
\hline & $\mathrm{n}$ & $\geq 3$ folds & $<3$ folds & $P$ & $\geq 3$ folds & $<3$ folds & $\mathrm{P}$ & $\geq 3$ folds & $<3$ folds & $\mathrm{P}$ \\
\hline \multicolumn{11}{|l|}{ Age } \\
\hline$<60$ & 50 & 33 & 17 & \multirow[t]{2}{*}{0.279} & 15 & 35 & \multirow[t]{2}{*}{0.413} & 12 & 38 & \multirow[t]{2}{*}{0.796} \\
\hline$\geq 60$ & 28 & 15 & 13 & & 6 & 22 & & 6 & 22 & \\
\hline \multicolumn{11}{|l|}{ Gender } \\
\hline Male & 50 & 32 & 18 & \multirow[t]{2}{*}{0.55} & 14 & 36 & \multirow[t]{2}{*}{0.774} & 15 & 35 & \multirow[t]{2}{*}{0.052} \\
\hline Female & 28 & 16 & 12 & & 7 & 21 & & 3 & 25 & \\
\hline \multicolumn{11}{|l|}{ Status } \\
\hline Dead & 50 & 35 & 15 & \multirow[t]{2}{*}{$0.040 *$} & 11 & 39 & \multirow[t]{2}{*}{0.19} & 10 & 40 & \multirow[t]{2}{*}{0.389} \\
\hline Alive & 28 & 13 & 15 & & 10 & 18 & & 8 & 20 & \\
\hline \multicolumn{11}{|l|}{ Histology type } \\
\hline Tubular type & 21 & 12 & 9 & \multirow[t]{3}{*}{0.549} & 7 & 14 & \multirow[t]{3}{*}{0.73} & 6 & 15 & \multirow[t]{3}{*}{0.78} \\
\hline Papillary type & 48 & 29 & 19 & & 23 & 36 & & 10 & 38 & \\
\hline Mixed type & 9 & 7 & 2 & & 2 & 7 & & 2 & 7 & \\
\hline \multicolumn{11}{|l|}{ Histology grade } \\
\hline Well differentiation & 34 & 15 & 19 & \multirow[t]{2}{*}{$0.005 *$} & 10 & 24 & \multirow[t]{2}{*}{0.663} & 7 & 27 & \multirow[t]{2}{*}{0.647} \\
\hline Moderate and poor & 44 & 33 & 11 & & 11 & 33 & & 11 & 33 & \\
\hline \multicolumn{11}{|l|}{ Stage } \\
\hline I, II & 25 & 10 & 15 & \multirow[t]{2}{*}{$0.007 *$} & 5 & 20 & \multirow[t]{2}{*}{0.344} & 4 & 21 & \multirow[t]{2}{*}{0.308} \\
\hline III, IV & 53 & 38 & 15 & & 16 & 37 & & 14 & 39 & \\
\hline \multicolumn{11}{|l|}{ Metastasis } \\
\hline No metastasis & 41 & 17 & 24 & \multirow[t]{2}{*}{$0.000 *$} & 13 & 28 & \multirow[t]{2}{*}{0.945} & 9 & 32 & \multirow[t]{2}{*}{0.804} \\
\hline Metastasis & 37 & 31 & 6 & & 12 & 25 & & 9 & 28 & \\
\hline
\end{tabular}




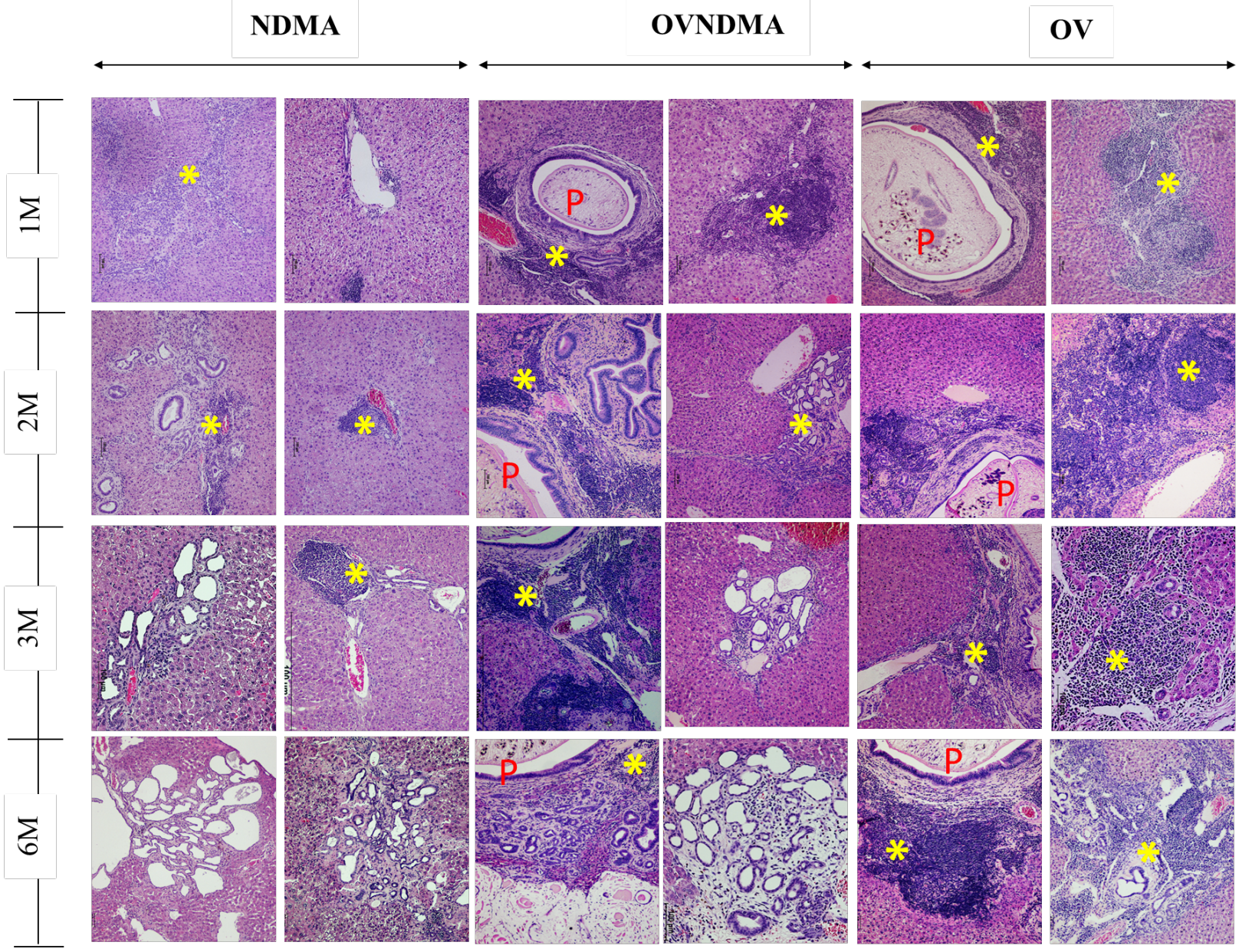

Figure 1. The Representative Histopathological of Hamster CCA Model
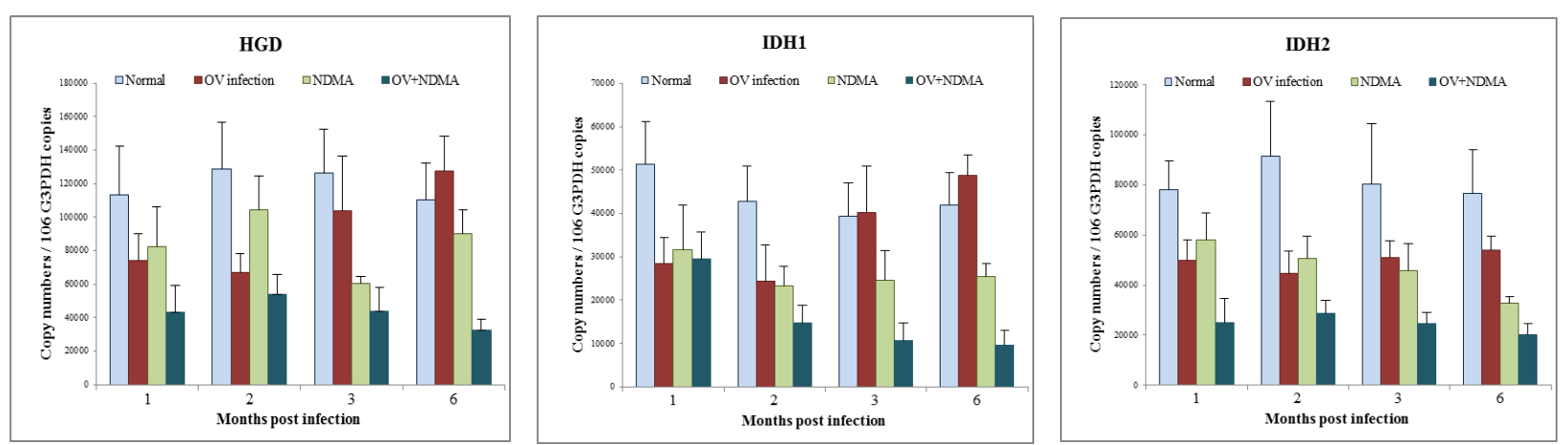

Figure 2. The Expressions of HGD, IDH1 and IDH2 in Hamster CCA Model

normal level at 3 and 6 months p.i. ( $>00.05)$. Much low level of expression in infection plus NDMA treatment was observed at all-time points $(\mathrm{p}<0.01)$. The expression was decreased slightly in the group of NDMA treatment alone, but much higher than those in the group of infection plus NDMA treatment. The expressions of both IDH1 and IDH2 were decreased in the group of infection plus NDMA treatment, as shown in (Figure 2). Infection only caused the decrease of expression of IDH1 at 1 and 2 months p.i., while there was no significant difference at 1 and 6 months p.i. The decreased expression of IDH2 was observed in the group of infection only at all time points.

\section{Expression of HGD, IDH1 and IDH2 in the human cases of $C C A$}

To confirm the expression of these 3 oxidative metabolism related genes in human CCA cases, we determined the expression of these genes in tumor tissue and adjacent tissue in same CCA case. Totally 78 cases were examined. The expression of HGD was decreased more than 2 folds in 55 cases (70.5\%), ranging from -2.1 to -50.5 folds with an average of -17.3 folds, and there was one case which showed the expression more than 2 folds. The expression of IDH1 was decreased more than 2 folds in 25 cases $(32.1 \%)$, ranging from -2.1 to -20.7 folds with an average of -8.1 folds, and there were 11 cases which showed the expression more than 2 folds $(14.1 \%)$. The expression of IDH1 was decreased more than 2 folds in 24 cases $(30.8 \%)$, ranging from -2.1 to -21.1 folds with an average of -7.9 folds, and there were 17 cases which showed the expression more than 2 folds $(21.8 \%)$ as shown in Figure 3. 

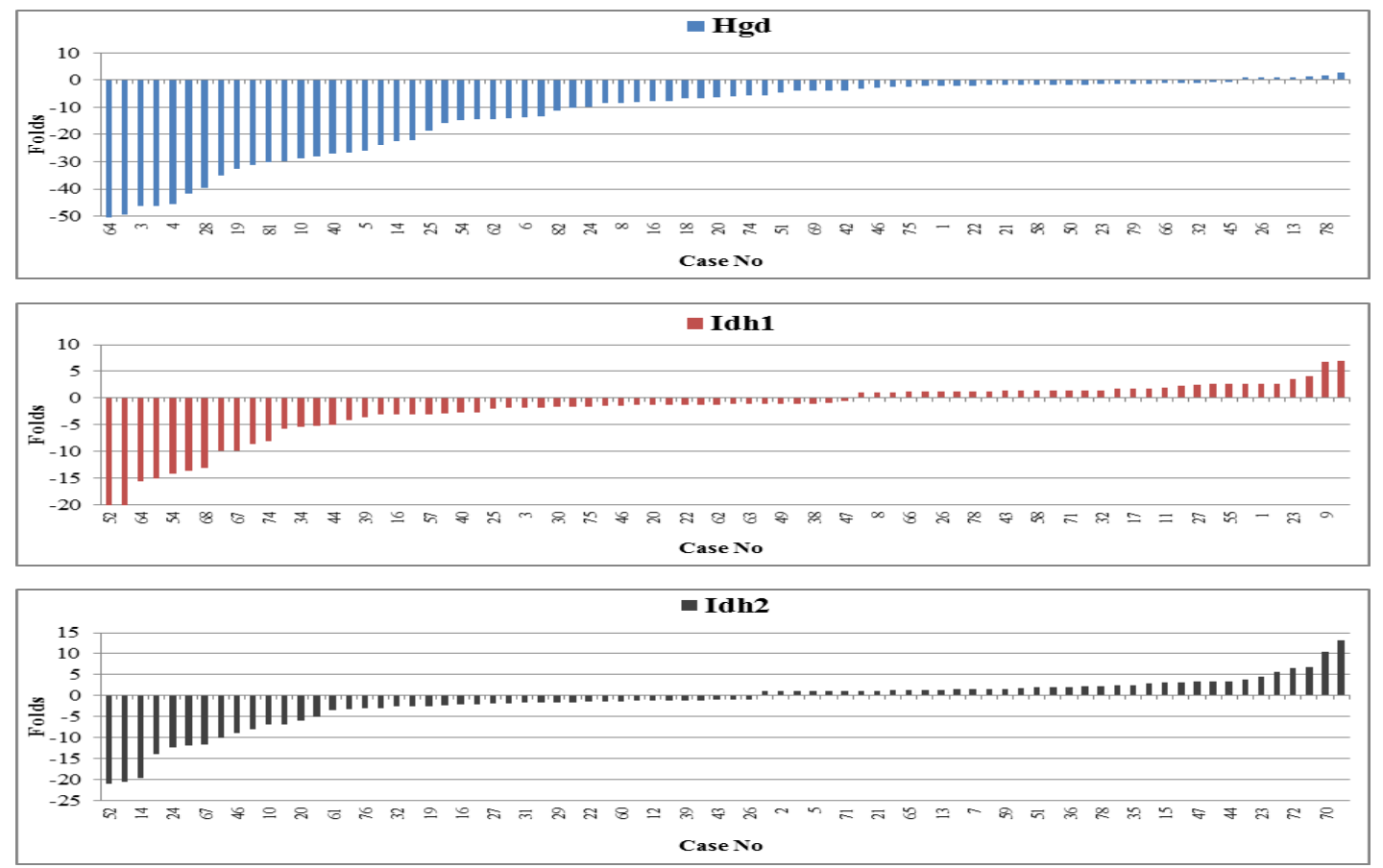

Figure 3. The Expressions of HGD, IDH1 and IDH2 in Human CCA
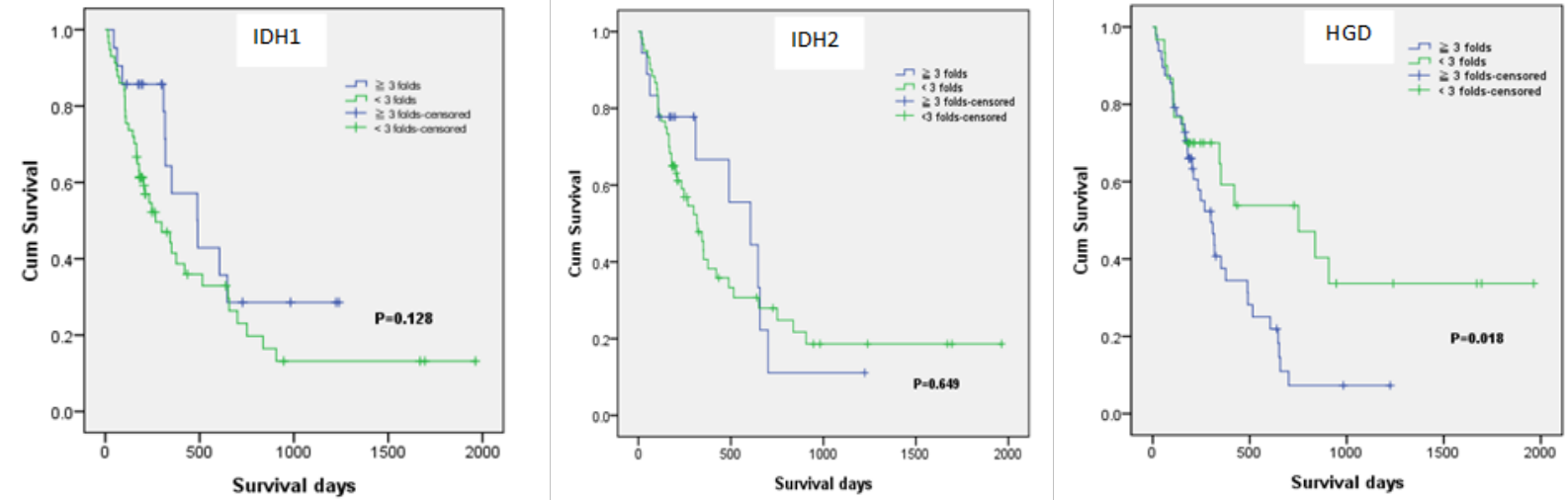

Figure 4. Survival Days

The correlation of expressions of HGD, IDH1 and IDH2 with clinicopathology

The correlation of HGD, IDH1 and IDH2 expressions at mRNA levels with all CCA patient's clinicopathological parameters was analyzed. As shown in Table 1, the expression levels were significantly correlated with the clinicopathology. Those patients with high HGD mRNA expression ( $\geq 3$ folds) showed more dead $(\mathrm{p}=0.040)$, more severe histopathology at grade III and IV $(p=0.005)$, advanced stage of CCA $(p=0.007)$, high grading stage and metastasis $(\mathrm{p}=0.000)$ than those with low expression ( $<3$ folds). IDH1 and IDH2 expression had no clinicopathological relation with age, gender, status, histological type, histological grading, staging and metastasis.

\section{Survival time}

Figure 4 showed the down or up regulation of IDH1 and IDH 2 had no effect on the survival rate but down regulation of HGD in human CCA shows that $<3$ fold down regulation of the HGD had higher survival rate than others.

\section{Discussion}

In animal model, O. viverrini infection induces inflammation cells filtration in the tissues around parasite. The inflammation is most obvious at 1-2 months post infection whereas the kinetic expression of all genes in infection animal and NDMA treatment group were not corresponding to the inflammation situation (Figure.1 and 2). However, the three IDH isoenzymes are important play role in the electrons exchange of metabolites between the mitochondria and the cytosol for cellular defense against oxidative damage as a source of NADPH. IDH1 and IDH2 found in cytoplasm and mitochondria respectively. Both IDH1 and IDH2 are important for shuttling electrons between the mitochondria and the cytosol (Sazanov et al., 1994; Minard et al., 1999; Joseph et al., 2006; Reitman et al., 2011). Recently, IDH1 and IDH2 are suppose to be the proto-oncogene (Reitman et al., 2011) so several reports show that in various cancers found IDH1 and IDH2 mutation such as acute myeloid leukemia (Dang et al., 2010), central chondrosarcoma and central and periosteal chondromas (Amary et al., 2011), prostate carcinoma (Kang et al., 2009), colorectal cancer (Sjöblom 
et al., 2006) and there are some reports show that found IDH1 and IDH2 mutation was good prognosis and high survival rate for glioblastomas (Hartmann et al., 2009; Yan et al., 2009; SongTao et al., 2012). Moreover, Borger et al. (2012) found $23 \%$ of the mutation of IDH1 and IDH2 in intrahepatic cholangiocarcinoma. According to above reasons thus, IDH1 and IDH2 expressions in normal condition or uninfected normal hamster control was remained in high level in all time points whereas in abnormal conditions, $O$. viverrini infection, NDMA administration and $O$. viverrini infection plus NDMA, were decreased (Figure 2). It may cause of the IDH1 and IDH2 mutation and electron transport abnormality in the liver including intrahepatic cholangiocarcinoma (Jiao et al., 2013) and mutated non- $O$. viverrini related CCA (Chan-On et al., 2013). HGD gene is homogentisate 1, 2-dioxygenase that provides instructions for making an enzyme called homogentisate oxidase, which is generally active in the liver and kidneys. This enzyme involve in a step-wise process that breaks down two protein building amino acids, phenylalanine and tyrosine, which play a role in making certain hormones, pigments, and brain chemicals called neurotransmitters. Most of these mutations change single amino acids used to build the homogentisate oxidase enzyme by changing its structure. There are reported that HGD mutations found in alkaptonuria (Al-sbou et al., 2012). This is the first report of down regulation of HGD in human and hamster CCA and moreover, the present data shows that $<3$ fold down regulation of the HGD had higher survival rate than others (Figure 3). The present study suggests that the animal CCA models induced by liver fluke and NDMA is correlated to the human CCA. The down regulation of IDH1, IDH2 and HGD genes was observed in both hamster CCA development at 1,2 and 3 months p.i. and the lowest expression was observed at 6 months p.i. (Figure 2). This was similar in most human CCA cases evidenced by down regulation of IDH1, IDH2 and HGD genes (Figure 3). Even through, the IDH1, IDH2 and HGD expression in group of infected $O$. viverrini alone or NDMA alone are not involved in any liver inflammations, induced by the $O$. viverrini infection or NDMA but also may involve in the structure change (mitochondria and cytosol) of hepatocyte which may involve in cholangiocarcinogenesis and be useful for CCAdiagnosis however, further study is needed.

\section{Acknowledgements}

This work was supported by a Grant-in-Aid for Scientific Research (21590463) from the Ministry of Education, Culture, Sports, Science and Technology of Japan; and Higher Education Research Promotion and National Research University Project of Thailand, Office of the Higher Education Commission, through the Health Cluster (SHep-GMS), Khon Kaen University and the Thailand research fund through Senior Research Scholar Grant no. RTA5580004. We also are grateful to the Department of Parasitology, the Liver Fluke and Cholangiocarcinoma Research Center, the Animal Experimental Unit, the Faculty of Medicine, Khon Kaen University.

\section{References}

Amary MF, Bacsi K, Maggiani F, et al (2011). IDH1 and IDH2 mutations are frequent events in central chondrosarcoma and central and periosteal chondromas but not in other mesenchymal tumours. J Pathol, 224, 334-43.

Al-sbou M (2012). Novel mutations in the homogentisate 1,2 dioxygenase gene identified in Jordanian patients with alkaptonuria. Rheumatol Int, 32, 1741-6.

Boonjaraspinyo S, Wu Z, Boonmars T, et al (2012). Overexpression of PDGFA and its receptor during carcinogenesis of Opisthorchis viverrini associated cholangiocarcinoma. Parasitol Int, 61, 145-50.

Boonmars T, Srirach P, Kaewsamut B, et al (2008). Apoptosisrelated gene expression in hamster opisthorchiasis post praziquantel treatment. Parasitol Res, 102, 447-55.

Boonmars T, Boonjaraspinyo S, Kaewsamut B (2009a). Animal models for Opisthorchis viverrini infection. Parasitol Res, 104, 701-3.

Boonmars T, Wu Z, Boonjaruspinyo S, et al (2009b). Alterations of gene expression of RB pathway in Opisthorchis viverrini infection-induced cholangiocarcinoma. Parasitol Res, 105, 1273-81.

Boonmars T, Wu Z, Boonjaruspinyo S, et al (2011). Involvement of c-Ski oncoprotein in carcinogenesis of cholangiocacinoma induced by Opisthorchis viverrini and N-nitrosodimethylamine. Pathol Oncol Res, 17, 219-27.

Borger DR, Tanabe KK, Fan KC, et al (2012). Frequent mutation of isocitrate dehydrogenase (IDH) 1 and IDH 2 in cholangiocarcinoma identified through broad-based tumor genotyping. Oncologist, 17, 72-9.

Chelikani P, Fita I, Loewen PC (2004). Diversity of structures and properties among catalases. Cell Mol Life Sci, 61, 192-208.

Chan-On W, Nairismägi ML, Ong CK, et al (2013). Exome sequencing identifies distinct mutational patterns in liver fluke-related and non- infection-related bile duct cancers. Nat Genet, 45, 1474-8.

Dang L, Jin S, Su SM (2010). IDH mutations in glioma and acute myeloid leukemia. Trends Mol Med, 16, 387-97.

Hayes JD, Flanagan JU, Jowsey IR (2005). Glutathione transferases. Annu Rev Pharmacol Toxicol, 45, 51-88.

Hartmann C, Meyer J, Balss J, et al (2009). Type and frequency of IDH1 and IDH2 mutations are related to astrocytic and oligodendroglial differentiation and age: a study of 1,010 diffuse gliomas. Acta Neuropathol, 118, 469-74.

IARC (1994). Infection with liver flukes (Opisthorchis viverrini, Opisthorchis felineus and Clonorchis sinensis). IARC Monogr Eval Carcinog Risks Hum, 61, 121-75.

IARC (2011). A Review of Human Carcinogens Part B: Biological Agents (Opisthorchis viverrini and Clonorchis sinensis). IARC Monogr Eval Carcinog Risks Hum, 100, 347-76.

Jiao Y, Pawlik TM, Anders RA, et al (2013). Exome sequencing identifies frequent inactivating mutations in BAP1, ARID1 A and PBRM1 in intrahepatic cholangiocarcinomas. Nat Genet, 45, 1470-3.

Jongsuksuntigul P, Imsomboon T (2003). Opisthorchiasis control in Thailand. Acta Trop, 88, 229-32.

Joseph JW, Jensen MV, Ilkayeva O, et al (2006). The mitochondrial citrate/isocitrate carrier plays a regulatory role in glucose-stimulated insulin secretion. J Biol Chem, 24, 35624-32.

Kang MR, Kim MS, Oh JE, et al (2009). Mutational analysis of IDH1 codon 132 in glioblastomas and other common cancers. Int J Cancer, 15, 353-5.

Kawanishi S, Hiraku Y (2006). Oxidative and nitrative DNA 
damage as biomarker for carcinogenesis with special reference to inflammation. Antioxid Redox Signal, 8, 1047-58

Kim W, Liau LM (2012). IDH Mutations in human glioma. Neurosurg Clin N Am, 23, 471-80.

McCord JM, Fridovich I (1969). Superoxide dismutase. An enzymic function for erythrocuprein (hemocuprein). $J$ Biol Chem, 25, 6049-55.

Miki H, Funato Y (2012). Regulation of intracellular signalling through cysteine oxidation by reactive oxygen species. $J$ Biochem, 151, 255-61.

Minard KI, McAlister-Henn L (1999). Dependence of peroxisomal beta-oxidation on cytosolic sources of NADPH. J Biol Chem, 5, 3402-6.

Pinlaor S, Hiraku Y, Ma N, et al (2004b). Mechanism of NO-mediated oxidative and nitrative DNA damage in hamsters infected with Opisthorchis viverrini: a model of inflammation-mediated carcinogenesis. Nitric Oxide, 11, 175-83.

Pinlaor S, Ma N, Hiraku Y, et al (2004a). Repeated infection with Opisthorchis viverrini induces accumulation of 8-nitroguanine and 8-oxo-7, 8-dihydro-2'-deoxyguanine in the bile duct of hamsters via inducible nitric oxide synthase. Carcinogenesis, 25, 1535- 42.

Qi ST, Yu L, Lu YT, et al (2011). IDH mutations occur frequently in Chinese I glioma patients and predict longer survival but not response to concomitant chemoradiotherapy in anaplastic gliomas. Oncol Rep, 26, 1479-85.

Raza H (2011). Dual localization of glutathione S-transferase in the cytosol and mitochondria: implications in oxidative stress, toxicity and disease. FEBS J, 278, 4243-51.

Reitman ZJ, Yan H (2011). Isocitrate dehydrogenase 1 and 2 mutations in cancer: alterations at a crossroads of cellular metabolism. J Natl Cancer Inst, 7, 932-41.

Sithithaworn P, Haswell-Elkins M (2003). Epidemiology of Opisthorchis viverrini. Acta Trop, 88,187-94.

Sriamporn S, Pisani P, Pipitgool V, et al (2004). Prevalence of Opisthorchis viverrini infection and incidence of cholangiocarcinoma in Khon Kaen, Northeast Thailand. Trop Med Int Health, 9, 588-94.

Srianujata S, Tonbuth S, Bunyaratvej S, et al (1984). High urinary excretion of nitrate and $\mathrm{N}$-nitrosoproline in opisthorchiasis subjects. IARC Sci Publ, 84, 544-6.

Sripa B, Pairojkul C (2008). Cholangiocarcinoma: lessons from Thailand. Curr Opin Gastroenterol, 24, 349-56.

Srivatanakul P, Ohshima H, Khlat M, et al (1991). Opisthorchis viverrini infestation and endogenous nitrosamines as risk factors cholangiocarcinoma in Thailand. Int J Cancer, $\mathbf{3 0}$, 821-5.

Sazanov LA, Jackson JB (1994). Proton-translocating transhydrogenase and NAD- and NADP-linked isocitrate dehydrogenases operate in a substrate cycle which contributes to fine regulation of the tricarboxylic acid cycle activity in mitochondria. FEBS Lett, 16, 109-16.

Sjöblom T, Jones S, Wood LD, et al (2006). The consensus coding sequences of human breast and colorectal cancers. Science, 13, 268-74.

SongTao Q, Lei Y, Si G, et al (2012). IDH mutations predict longer survival and response to temozolomide in secondary glioblastoma. Cancer Sci, 103, 269-73.

Thamavit W, Bhamarapravati N, Sahaphong S, et al(1987).Effects of dimethylnitrosamine on induction of cholangiocarcinoma in Opisthorchis viverrini-infected Syrian golden hamsters. Cancer Res, 38, 4634-63.

Thamavit W, Pairojkul C, Tiwawech D, et al (1993). Promotion of cholangiocarcinogenesis in the hamster liver by bile duct ligation after dimethylnitrosamine initiation.Carcinogenesis, 14, 2415-7.
Valko M, Leibfritz D, Moncol J, et al (2007). Free radicals and antioxidants in normal physiological functions and human disease. Int J Biochem Cell Biol, 39, 44-84.

Wu Z, Boonmars T, Boonjaraspinyo S, et al (2011). Candidate genes involving in tumorigenesis of cholangiocarcinoma induced by Opisthorchis viverrini infection. Parasitol Res, 109, 657-73.

Yan H, Parsons DW, Jin G, et al (2009). IDH1 and IDH2 mutations in gliomas. $N$ Engl J Med, 19, 765-73 